\title{
Effects of Bifidobacterium bifidum in Mice Infected with Citrobacter rodentium
}

\author{
Bijun Wen ${ }^{1}$, Amel Taibi ${ }^{1}$, Christopher R. Villa ${ }^{1}$, Shin-Hann Lee ${ }^{1}$, Sofia Sagaidak ${ }^{1}$ \\ and Elena M. Comelli $1,2, * \mathbb{D}$ \\ 1 Department of Nutritional Sciences, Faculty of Medicine, University of Toronto, Toronto, ON M5S 1A8, \\ Canada; bijun.wen@mail.utoronto.ca (B.W.); amel.taibi@utoronto.ca (A.T.); \\ christopher.villa@mail.utoronto.ca (C.R.V.); hann.lee@mail.utoronto.ca (S.-H.L.); \\ sofia.sagaidak@mail.utoronto.ca (S.S.) \\ 2 Joannah and Brian Lawson Centre for Child Nutrition, Faculty of Medicine, University of Toronto, Toronto, \\ ON M5S 1A8, Canada \\ * Correspondence: elena.comelli@utoronto.ca
}

Received: 12 December 2018; Accepted: 11 February 2019; Published: 14 February 2019

\begin{abstract}
In vitro and in vivo studies suggest that selected Bifidobacterium bifidum strains sustain intestinal homeostasis. This study aimed to examine whether the administration of B. bifidum MIMBb75 (BB75) attenuates Citrobacter rodentium infection, a murine model for enteric infection and inflammatory bowel disease in humans. C57B16/J mice were randomized to receive BB75 daily starting before or after $C$. rodentium infection. BB75 load and infection kinetics were monitored. On day 10 post-infection (p.i.), histological parameters of the large intestine were assessed. Barrier integrity was evaluated by pathogen translocation to secondary organs and in vivo permeability test. Fecal C. rodentium load peaked at $10^{10} \mathrm{CFU} / \mathrm{g}$ at day 10 p.i., with clearance at day 24 p.i., regardless of probiotic treatment. BB75 administration resulted in $10^{7}$ cells $/ \mathrm{g}$ of feces with no effect of timing of administration. BB75 treatment did not attenuate $C$. rodentium-induced crypt hyperplasia nor inflammation. C. rodentium and BB75 can co-exist in the gut with no mutual displacement. However, BB75 cannot counteract $C$. rodentium pathology. Our findings provide insight for the understanding of probiotics behavior and their clinical relevance in intestinal inflammation.
\end{abstract}

Keywords: Bifidobacterium bifidum MIMBb75; Citrobacter rodentium; inflammatory bowel disease (IBD); EHEC infection; colitis; probiotics

\section{Introduction}

Bifidobacterium bifidum is a Gram-positive anaerobe belonging to the Actinobacteria phylum. B. bifidum is one of the first colonizers of the infant gut [1] and members of this species have many health-promoting effects, including immunomodulation [2], bacteriocin production [3], pathogen exclusion [4], and maintenance of health-compatible microbiota [5]. These beneficial effects may have implications in intestinal acute and chronic inflammation, including following enteric infection or in inflammatory bowel disease (IBD). For instance, in vitro, B. bifidum LMG13195 sustains the integrity of HT29 cells monolayers [6] and B. bifidum ATCC 29521 interferes with attachment and colonization of the foodborne pathogen enterohemorrhagic Escherichia coli (EHEC) [7]. In vivo, B. bifidum BGN4 alleviates lymphocytes infiltration and Th1-type cytokines production in a naive T-cell transfer mouse model of IBD [8]. B. bifidum S17 [9] and, more recently, B. bifidum PI22 [10] were shown to dampen inflammation in a trinitrobenzene sulfonic acid-induced colitis mouse model.

B. bifidum MIMBb75 (BB75) is a human isolate [11] and a probiotic that exhibits strong adhesive properties to intestinal epithelial cells [12], mitigating intestinal discomfort in patients with 
irritable bowel syndrome [13]. We found that, when administered to mice at $10^{8}$ colony-forming units (CFU), BB75 is recovered all along the large intestine, with a preference for its proximal regions [14]. BB75 induces intestinal responses at the gene expression level and has immunomodulatory properties [15,16]. In Caco-2 cells, BB75 downregulates expression of EPAS1 [15], which is a transcription factor activating inflammation during infectious colitis [17]. Another in vitro study reported that the BB75 surface murein lytic enzyme TgaA could induce dendritic cell activation and IL-2 production [16]. In this study, we investigate the ability of BB75 to prevent and/or mitigate intestinal inflammation in mice infected with Citrobacter rodentium. C. rodentium is a murine enteric pathogen that closely resembles human EHEC pathogenesis and can induce colitis via a Th1/Th17-dominated inflammatory response in the mouse intestine [18]. Thus, C. rodentium infection is commonly used as a model for enteric infection and IBD [18]. Probiotic Lactobacillus strains and B. breve UCC2003 were previously found to mitigate colonic crypt hyperplasia and mucosal inflammation in this model [19-25]. However, studies focused on the effects of other bifidobacteria species and strains on pathologies associated with $C$. rodentium infection are lacking. It is anticipated that our study will contribute to the growing evidence on the role of probiotics in intestinal inflammation.

\section{Materials and Methods}

\subsection{Mice and Bacteria}

Eighty specific pathogen-free C57Bl6/J male mice, six weeks of age, were obtained from Jackson Laboratories (Bar Harbor, ME, USA) and housed in a containment unit (biosafety level 2), where they received sterile chow and water ad libitum. C. rodentium DBS100 was generously provided by Dr. Dana Philpott, University of Toronto (originally obtained from Dr. David Schauer). B. bifidum MIMBb75 was used as previously described [14]. Animal study design and procedures were approved by the animal ethics committee at the University of Toronto (Animal Use Protocol Number: 20010228) and were in accordance with the Regulations of the Animals for Research Act in Ontario and the Guidelines of the Canadian Council on Animal Care.

\subsection{Study Design}

Mice were randomized into four groups ( $\mathrm{n}=20$ /group): (1) Sham infected, (2) C. rodentium infected (CR), (3) C. rodentium infected with daily administration of B. bifidum initiated $5 \mathrm{~h}$ after, but on the same day as the infection (BB-CR), (4) C. rodentium infected with daily B. bifidum administration initiated one week (7 days) before the infection (BB pre-CR) (Figure 1a). Infection was performed by intra-gastric gavage of $100 \mu \mathrm{L} \mathrm{LB}$ (lysogeny broth)-cultured C. rodentium $\left(10^{9} \mathrm{CFU} / \mathrm{mL}\right.$ ) or an equal volume of sterile LB (Sham), as previously described [26]. Daily B. bifidum treatment was performed by intra-gastric gavage of $200 \mu \mathrm{L}$ B. bifidum suspension in PBS $\left(10^{9} \mathrm{CFU} / \mathrm{mL}\right)$ or an equal volume of sterile PBS.

Body weights were measured and freshly passed fecal pellets were collected on post-infection (p.i.) days 2, 4, 6, 8, 9 and just before sacrifice. A subset of mice ( $\mathrm{n}=16$ /group) were sacrificed on day 10 p.i., which is the peak of infection [27], by cervical dislocation after brief exposure to carbon dioxide; caecum (the initial site of infection about 2-3 days p.i.) and distal colon (the major site of infection at day 10 p.i. and defined as the distal $3.5 \mathrm{~cm}$ of the colon after excision of the rectum), kidneys, spleen and liver were dissected on ice and fixed in $10 \%$ formalin for histology or used for C. rodentium quantification as explained below. Four mice per group were retained to monitor the effect of BB75 on C. rodentium clearance after day 10 p.i. and thereafter, freshly passed fecal pellets were collected every other day until reaching clearance (below the detection limit for two consecutive days), as previously described $[27,28]$. 


\subsection{Bacteria Culturing and Quantification}

For gavage, C. rodentium DBS100 was grown in lysogeny broth (Luria-Bertani, LB) aerobically at $37^{\circ} \mathrm{C}$ for $16 \mathrm{~h}$, as previously described [26]. Viable counts of $C$. rodentium in liquid culture, gavage suspension, feces, liver and spleen were determined by classical culturing on MacConkey Agar (BioShop); after aerobic incubation for $24 \mathrm{~h}$ at $37^{\circ} \mathrm{C} ; \mathrm{C}$. rodentium colonies were identified based on morphology with a detection limit of $10^{3} \mathrm{CFU} / \mathrm{g}$ [19].

B. bifidum MIMBb75 was grown in an anaerobic chamber (Coy Laboratory Products, Grass Lake, MI, USA) at $37^{\circ} \mathrm{C}$ in Man Rogosa Sharpe broth, supplemented with $0.05 \%$ L-cysteine hydrochloride (cMRS) for $24 \mathrm{~h}$. The culture was washed and re-suspended in sterile pre-reduced PBS $(200 \mu \mathrm{L})$ immediately before gavage. Viable counts of B. bifidum in gavage culture were enumerated throughout the study with a hemacytometer and by classical culturing on cMRS agar to confirm viability. To assess the fecal load of B. bifidum, fecal pellets were collected from uninfected mice 2 days post-B. bifidum initiation (i.e., BB pre-CR) and from mice in all groups on day 9 p.i. DNA was extracted using the Omega E.Z.N.A.TM Stool DNA Isolation Kit (Omega Bio-Tek, Norcross, GA), modified as previously described [14]. Quantitative Real-Time PCR was performed with $50 \mathrm{ng}$ of DNA using the SYBR Green Master Mix (Applied Biosystems, Carlsbad, CA) with specific primers targeting the bopA gene, which is specific to the B. bifidum species [11] (Forward: 5'ACCGAATTCGCCTGTCACTT3'; Reverse: $5^{\prime}$ ACGGCGCGGATTCGT3'). Each reaction was run in triplicates in a 384-well optical plate using a 7900 HT Real-Time PCR machine (Applied Biosystems) with default conditions. Bacterial counts were determined using pre-constructed standard curves, and data were expressed as log 10 cells per gram of feces.

\subsection{Fluorescein Isothiocyanate-Dextran In Vivo Permeability Assay}

On day 10 p.i., intestinal permeability was assessed in a subset of overnight fasted mice ( $\mathrm{n}=8$ /group) using the fluorescein isothiocyanate (FITC)-dextran (4 kDa, Sigma-Aldrich, Oakville, Canada) assay [26,29]. Serum FITC-dextran concentration was quantified by fluorometry (FusionTM, PerkinElmer) with an excitation wavelength of $485 \mathrm{~nm}$ and emission wavelength of $535 \mathrm{~nm}$ [26,29].

\subsection{Histological Analysis}

Hematoxylin and Eosin-stained $5 \mu \mathrm{m}$ tissue sections were used to score inflammation on a scale of $0-4$, as previously described [29,30]. A minimum of 1 transverse and 2 longitudinal sections per cecum and distal colon tissue, and 5 far-separated fields per section were assessed to determine the mean mucosal damage score and crypt depths for each mouse with the examiner blinded to the various groups [26,29].

\subsection{Statistical Analysis}

C. rodentium fecal counts and body weight changes were analyzed using repeated-measures analysis of variance (ANOVA). Differences in organ weights, crypt hyperplasia, colon damage scores, intestinal permeability, and bacterial translocation were determined by one-way ANOVA followed by the Bonferroni's post-hoc test as necessary. The difference of fecal B. bifidum counts before and after infection and between B. bifidum-treated groups were assessed by Student's t-test. A P-value of $\mathrm{P}<0.05$ was considered statistically significant. All statistical analyses were performed with Prism 7.0d software (GraphPad Software, La Jolla, CA, USA).

\section{Results}

\subsection{C. rodentium Infection Kinetics and Characteristics}

C. rodentium was detectable in the feces two days after infection in all infected groups; viable counts increased until reaching a plateau $\left(10^{10} \mathrm{CFU} / \mathrm{g}\right)$ at day 10 p.i. (Figure $\left.1 \mathrm{~b}\right)$, then decreased 
rapidly and reached clearance on approximately day 24 p.i. on average, with no significant differences among groups. The infection did not result in significant changes of body weight among groups (Figure 1c). Spleen weights were significantly higher in the infected groups compared to the Sham but did not differ among treatments (CR: $0.54 \pm 0.075$, BB-CR: $0.61 \pm 0.06$, BB pre-CR: $0.58 \pm 0.07$, Sham: $0.28 \pm 0.02 \%$ body weight; $\mathrm{p}<0.01$ ); there was no significant difference in kidney and liver weights among groups (Figure 1d).

a.

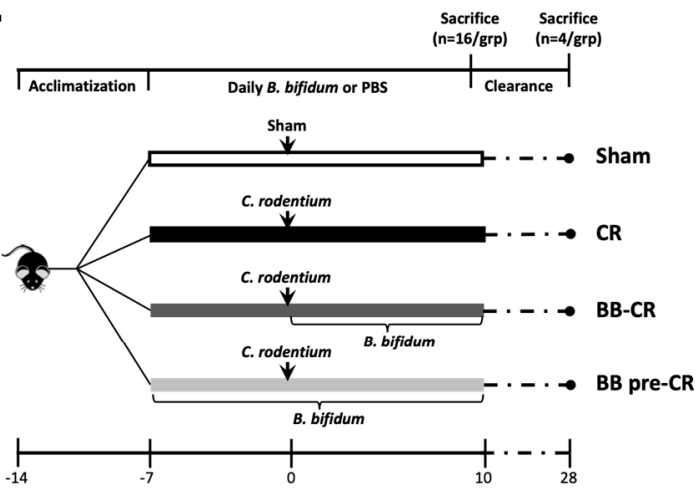

c.

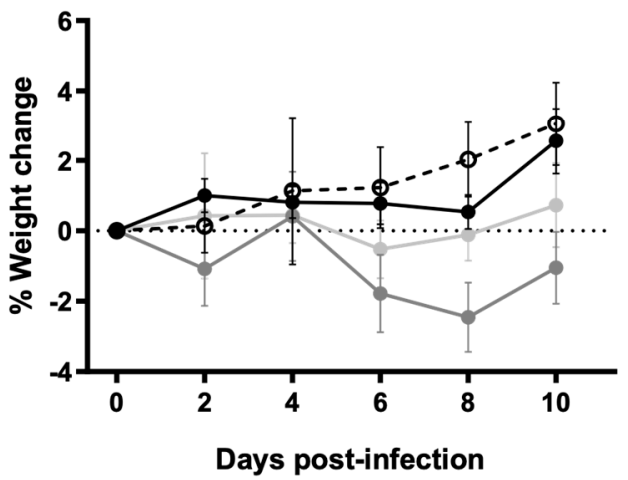

b.

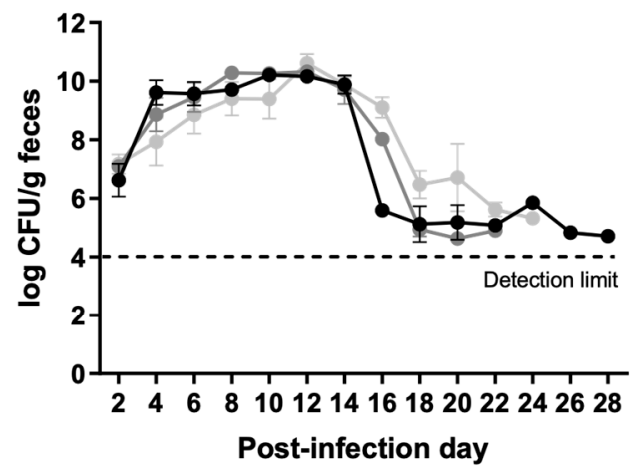

d.

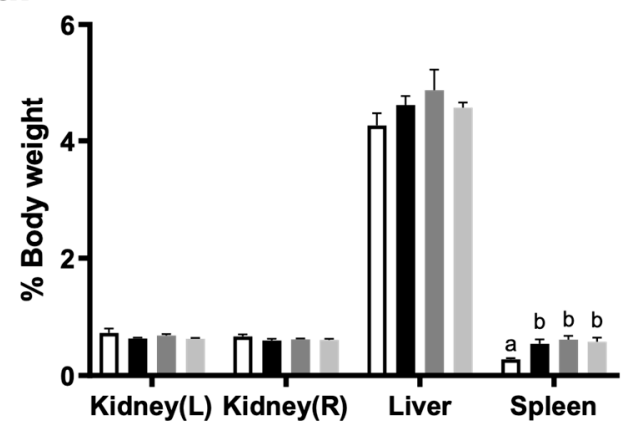

Figure 1. Study design and C. rodentium infection model (a) study design; (b) viable C. rodentium counts in infected mice feces were quantified every other day p.i., $\mathrm{n}=9-13$ /group (day 2-10 p.i.), and $n=1-4$ /group (day 12-28 p.i.); (c) percentage of body weight change among groups after infection, $\mathrm{n}=12$ /group; (d) percentage of organ weight per gram of body weight among Sham and infected groups on day 10 p.i., $n=8$ /group. Data are presented as mean \pm SE. Statistical significance was determined by repeated-measures ANOVA for $C$. rodentium counts and weight change and one-way ANOVA for organ weight followed by Bonferroni's post-hoc test. Different superscripts $(a, b)$ indicate

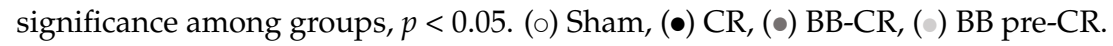

\subsection{B. bifidum Recovery in Feces}

Before infection, B. bifidum load in feces of the BB pre-CR mice after day 2 of administration was $7.2 \pm 0.13 \mathrm{log}$ cell $/ \mathrm{g}$ and there was no significant difference in B. bifidum load before $(-5$ p.i.) and after infection (9 p.i.) in this group. On day 9 p.i, there was also no significant difference in B. bifidum load between the two probiotic-treated groups (BB-CR: 7.0 \pm 0.4 , BB pre-CR: $6.7 \pm 0.4 \mathrm{log}$ cell/g) (Figure 2). B. bifidum was not detected in CR and Sham mice, which did not receive B. bifidum treatment. 


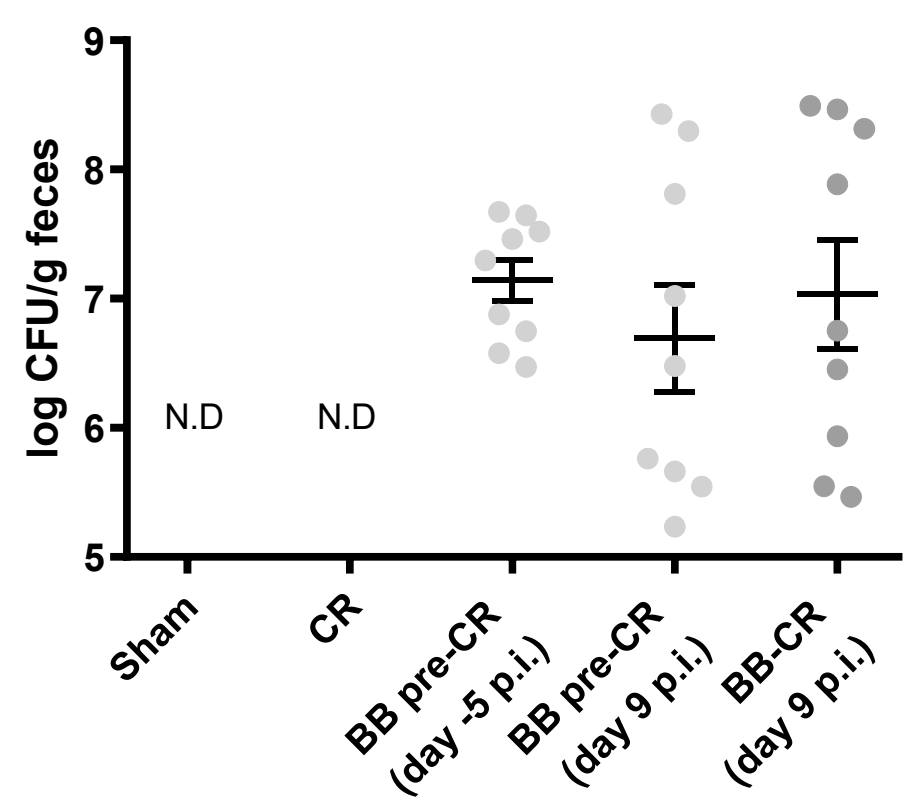

Figure 2. Fecal BB75 load before and after infection. Real-time PCR quantification of fecal BB75 in all groups of mice ( $n=9$ /group) after $C$. rodentium $(C R)$ infection, and in BB pre-CR group two days after initiation of BB75 (i.e., five days before infection). Statistical significance was determined by Student's t-test, data presented as mean \pm SE. N.D, not detectable.

\subsection{Intestinal Morphology and Histopathology}

Macroscopic observation of the large intestine morphology revealed that infected mice had enlarged cecum and loose intestinal content compared to the Sham; BB75 treated mice did not exhibit apparent differences compared to the untreated mice (Figure 3a). Histopathology analysis showed that $C$. rodentium infected mice exhibited a significant increase of crypt length in the cecum and distal colon, compared to the Sham (Figure 3b-d). Cecal crypt length of mice receiving BB75 did not differ significantly from Sham nor infected controls, suggesting a marginal but not significant normalization effect of BB75 in the cecum. However, distal colon crypt lengths of mice receiving BB75 were significantly greater than Sham control, but did not differ from infected controls. Histological analysis of the cecum and distal colon tissue revealed significant difference in tissue damage scores between infected mice and Sham mice, but no effect of B. bifidum administration (Figure $3 \mathrm{~b}, \mathrm{e}, \mathrm{f}$ ). 
a.

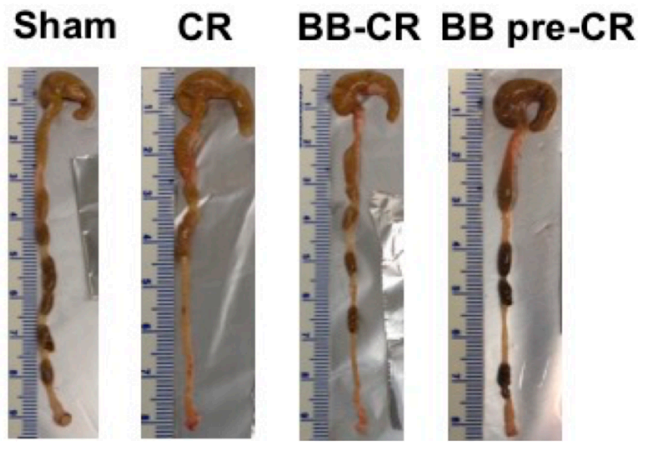

C.

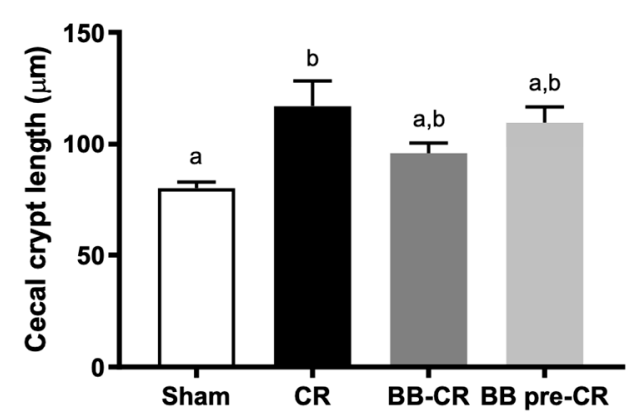

e.

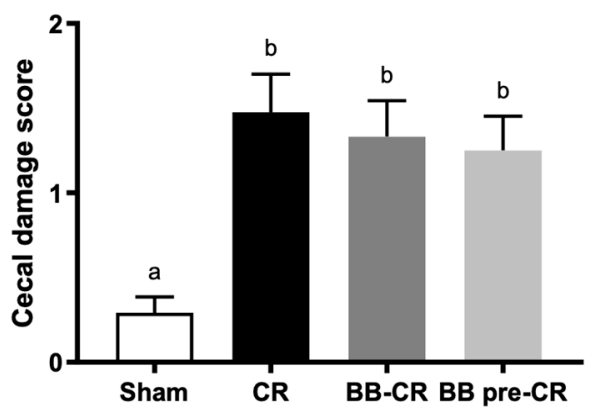

b.

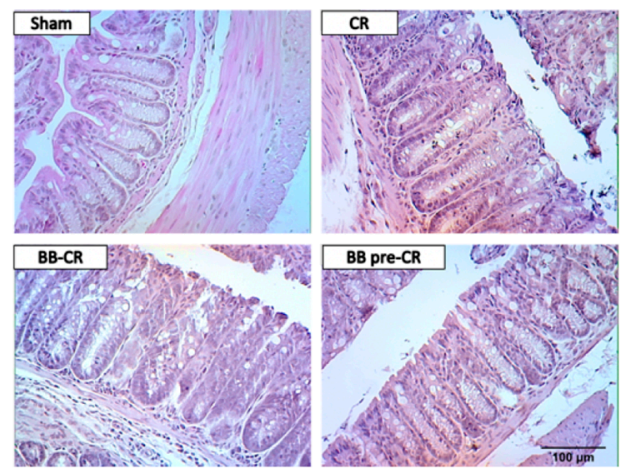

d.

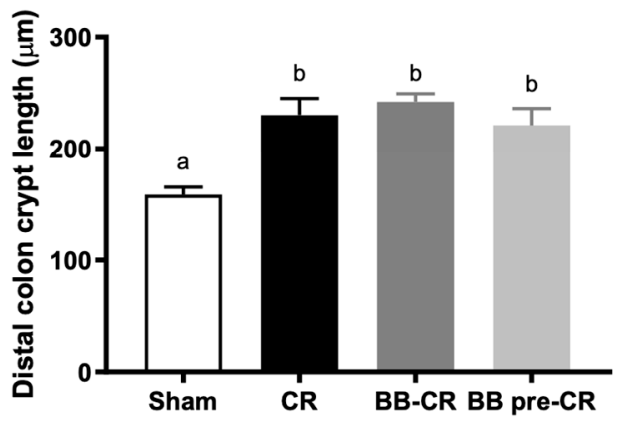

f.

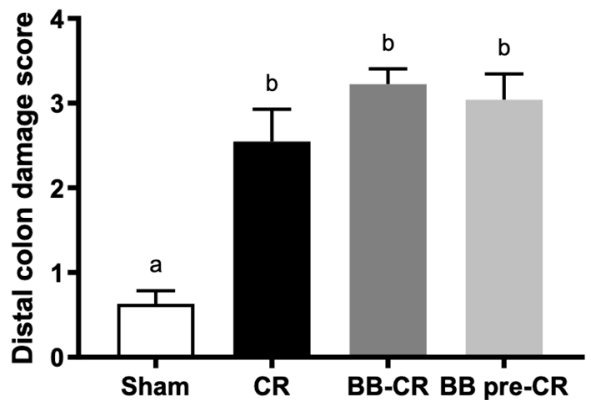

Figure 3. The effect of BB75 on intestinal crypt hyperplasia and tissue damage at day 10 p.i. (a) colon morphology; (b) representative H\&E stained histology of distal colon tissues on day 10 p.i.; (c) ceacal $(\mathrm{n}=7-8 /$ group) and (d) distal colon crypt lengths ( $\mathrm{n}=10 /$ group); (e) ceacal and (f) distal colon damage score ( $n=10$ /group) were accessed on a scale of $0-4(0$, no signs of inflammation; 1 , minimal evidence of inflammatory cell infiltration; 2 , significant evidence of inflammatory cell infiltration; 3 , significant evidence of inflammation with goblet cell depletion; 4 , sever inflammation characterized by widespread inflammatory cells infiltration, goblet cell depletion and destruction of the mucosal architecture). Results are expressed as mean \pm SE. Statistical significance was determined by one-way ANOVA, followed by the Bonferroni's post-hoc test. Different superscripts $(a, b)$ indicate statistical significance between groups, $p<0.05$.

\subsection{Barrier Integrity}

There was no difference in intestinal permeability measured by the FITC-dextran assay among the four groups on day 10 p.i. (Figure $4 a$ ). C. rodentium was quantifiable in the liver and spleen of infected but not control mice; though, B. bifidum treatment did not affect these counts (Figure $4 b, c)$. 
a.

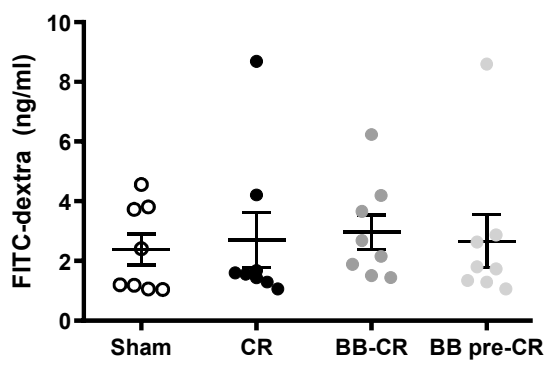

b.
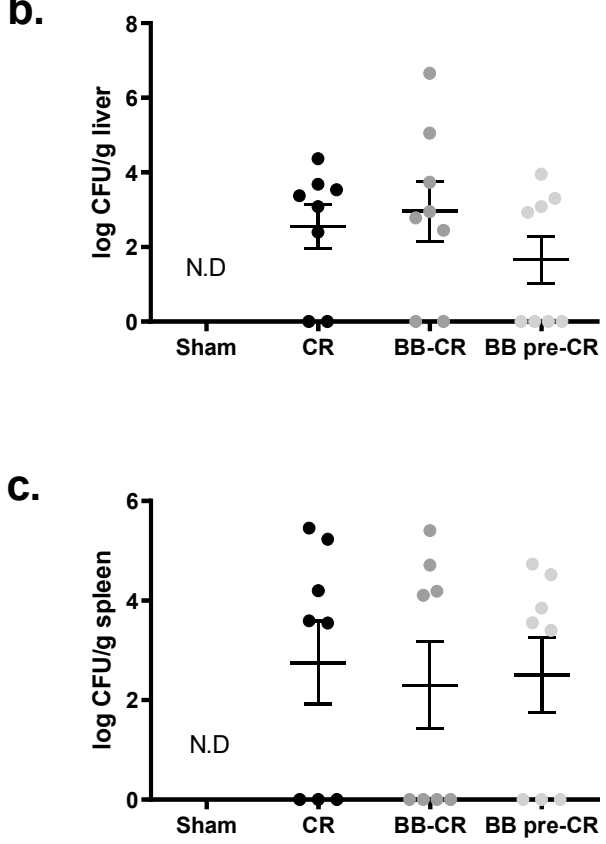

Figure 4. The effect of BB75 on the intestinal barrier at day 10 p.i. (a) In vivo FITC-dextran intestinal permeability test ( $\mathrm{n}=8$ /group); no difference was detected in intestinal permeability among the groups, despite $C$. rodentium or BB75 treatment; C. rodentium translocation to (b) liver and (c) spleen ( $\mathrm{n}=8$ /group); no difference in C. rodentium translocation was detected among groups. Results are presented as mean \pm SE. Statistical significance was determined by one-way ANOVA. N.D, not detectable.

\section{Discussion}

This study investigated the impact of probiotic B. bifidum MIMBb75 on C. rodentium infection. The probiotic could be recovered in the feces of the infected mice. Though, its administration in either a preventative or treatment manner did not improve intestinal pathology (C. rodentium colonization, crypt hyperplasia, inflammation, and barrier dysfunction).

BB75 was quantified in the feces at day 9 p.i. and counts were found to align with the fecal load in healthy mice [14]; moreover, fecal BB75 counts in BB pre-CR group before and after infection (Figure 2) were not significantly different. This indicates that infection did not interfere with B. bifidum fecal recovery. $C$. rodentium is only pathogenic in murine and can replace almost $90 \%$ of the resident microbiota [18,31]. The fact that BB75, a human-restricted strain, was not displaced, is in line with reports showing the excellent gut colonization capacity of this strain [11].

Selected probiotic bacteria were found to result in a reduction of $C$. rodentium load during infection [19-25]. For example, treatment of L. rhamnosus R0011 and L. acidophilus R0052 reduced C. rodentium attachment to T84 epithelial cells in vitro, and its load in colonic luminal contents at day nine p.i. in C57Bl/6 mice [19]. A three-day pre-treatment of B. breve UCC2003 in BALB/c mice 
reduced fecal C. rodentium load from day 3 to 14 p.i. [24]. In the present study, BB75 administration did not suppress $C$. rodentium colonization nor did it improve its clearance, suggesting that the two strains can co-exist in the gut ecosystem with no mutual exclusion. Since we previously showed that BB75 predominately localizes to the cecum rather than to the distal colon [14], where C. rodentium preferentially resides, future studies may examine colonization competition between the two bacteria in a region-specific manner.

Competition for colonization sites within the intestine is not always necessary for probiotics to mitigate C. rodentium pathology. For example, treatment with B. breve UCC2003 for three days prior to infection attenuated crypt hyperplasia without affecting $C$. rodentium colonization or $\mathrm{A} / \mathrm{E}$ lesion formation in C57Bl/ 6 mice [25]. A more recent study conducted by this group also found that administration of fermented product from Lactobacillus species to C57Bl/ 6 mice improved infection, but did not alter $C$. rodentium colonization kinetics [22]. Yet, in the present study, TMCH and histological damage of the cecum and distal colon were not attenuated in mice treated with BB75 whether before or after $C$. rodentium challenge. To our knowledge, no studies are available of B. bifidum in a C. rodentium-induced colitis model. It is known that the gene expression program of BB75 changes in response to different local environments. For example, the adhesion efficiency of BB75 was found to vary across various differentiation stages of epithelial cells [32]. It was also shown that the expression level of pili (adhesion factors) in other B. bifidum strains varies according to the available carbon source [33]. Thus, the ability of BB75 to attenuate infection may depend on the environmental conditions, and may differ in the human ecosystem. It is also possible that this species, or this particular strain, is not the optimal choice in this context. Finally, it is possible that the conditions chosen for this study mask benefits. We used a very well established and reproducible C. rodentium infection protocol [26,29], which has been employed in the past for probiotic studies [19-23]. However, we cannot exclude that BB75 may have beneficial effects in other colitis models. While we used live probiotic cells, which is in accordance with the current definition of probiotic [34], it is also possible that BB75 administered as a post or para-probiotic, may elicit different effects.

One unexpected observation in the present study was the general increase of intestinal permeability in all mice including those in the Sham-infected group with no differences among groups. It is known that $C$. rodentium infection results in intestinal barrier dysfunction and therefore, increased paracellular translocation of the macromolecular tracer FITC-dextran [21,29]. For example, Rodrigues et al. showed that on day 10 p.i. serum FITC-dextran concentration in infected adult mice was two-times higher than that of the Sham mice [21], which we have also observed in a recent study [29]. Surprisingly, serum FITC-dextran concentration of the Sham mice in the present study was as high as the infected groups on day 10 p.i, suggesting impaired barrier integrity in healthy uninfected mice. Mice in our study underwent daily intra-gastric gavage for 17 days. This technique allows to control for the amount of probiotic received by each mouse, but it imposes physical and psychological stress to the animals. Chronic psychological stress can trigger the release of the glucocorticoid hormone and lead to mucosal barrier dysfunction, thereby increased intestinal permeability and host defense mechanism impairment [35]. In fact, studies that showed positive effects of probiotic treatment with normal ranges of intestinal permeability in the Sham controls, administered probiotics daily in drinking water [19-21]. Other studies used prolonged daily gavage of probiotics, but did not measure permeability [25]. In line with this, C. rodentium translocation to secondary organs, spleen, and liver, was similar among the infected groups, indicating similar alteration of the barrier integrity.

\section{Conclusions}

In summary, this is the first study addressing the effects of BB75 in infectious colitis. It was found that administration of this strain did not attenuate C. rodentium colonization, intestinal inflammation, crypt hyperplasia and barrier dysfunction. Findings from this study may provide insights for future investigation, particularly with respect to the protocol in which probiotics are administered. From a translational perspective, this study may help in the selection of probiotics to be tested in the clinical 
setting [36,37], at least in the context of Crohn's disease, and provides further evidence that probiotics benefits cannot be extrapolated across different conditions.

Author Contributions: Conceptualization, B.W., A.T. and E.M.C.; methodology, B.W., A.T., C.R.V. and E.M.C.; formal analysis, B.W., S.-H.L., and S.S.; investigation, B.W., A.T., C.R.V., S.-H.L., and S.S.; writing-original draft preparation, B.W. and E.M.C.; writing-review and editing, B.W., A.T., C.R.V., S.-H.L., S.S., and E.M.C.; visualization, B.W.; supervision, E.M.C.

Funding: This study was supported by the Natural Sciences and Engineering Research Council of Canada (NSERC) (Grant \# RGPIN 356124-2013), the J. P. Bickell Foundation, and the James H. Cummings Foundation. Elena M. Comelli holds the Lawson Family Chair in Microbiome Nutrition Research at the University of Toronto. Bijun Wen was the recipient of a NSERC Alexander Graham Bell Canada Graduate Scholarship. Christopher Villa was partially funded by the Banting and Best Diabetes Centre -Novo Nordisk Studentship and Tamarack Graduate Award in Diabetes Research and by an Ontario Graduate Scholarship. Sofia Sagaidak was the recipient of a NSERC Undergraduate Student Research Award.

Acknowledgments: The authors would like to thank the Division of Comparative Medicine staff at the University of Toronto for their help with animal care.

Conflicts of Interest: EMC has received research support from Lallemand Health Solutions. The other authors have no conflict of interest.

\section{References}

1. Biavati, B.; Vescovo, M.; Torriani, S.; Bottazzi, V. Bifidobacteria: History, ecology, physiology and applications. Ann. Microbiol. 2000, 50, 117-131.

2. Ohno, H.; Tsunemine, S.; Isa, Y.; Shimakawa, M.; Yamamura, H. Oral administration of Bifidobacterium bifidum G9-1 suppresses total and antigen specific immunoglobulin E production in mice. Biol. Pharm. Bull. 2005, 28, 1462-1466. [CrossRef] [PubMed]

3. Yildirim, Z.; Winters, D.K.; Johnson, M.G. Purification, amino acid sequence and mode of action of bifidocin B produced by Bifidobacterium bifidum NCFB 1454. J. Appl. Microbiol. 1999, 86, 45-54. [CrossRef] [PubMed]

4. Miki, K.; Urita, Y.; Ishikawa, F.; Iino, T.; Shibahara-Sone, H.; Akahoshi, R.; Mizusawa, S.; Nose, A.; Nozaki, D.; Hirano, K.; et al. Effect of Bifidobacterium bifidum fermented milk on Helicobacter pylori and serum pepsinogen levels in humans. J. Dairy Sci. 2007, 90, 2630-2640. [CrossRef] [PubMed]

5. Chow, W.L.; Lee, Y.K. Free fucose is a danger signal to human intestinal epithelial cells. Br. J. Nutr. 2008, 99, 449-454. [CrossRef] [PubMed]

6. Lopez, P.; Gonzalez-Rodriguez, I.; Sanchez, B.; Ruas-Madiedo, P.; Suarez, A.; Margolles, A.; Gueimonde, M. Interaction of Bifidobacterium bifidum LMG13195 with HT29 Cells Influences Regulatory-T-Cell-Associated Chemokine Receptor Expression. Appl. Environ. Microbiol. 2012, 78, 2850-2857. [CrossRef] [PubMed]

7. Bayoumi, M.A.; Griffiths, M.W. In vitro inhibition of expression of virulence genes responsible for colonization and systemic spread of enteric pathogens using Bifidobacterium bifidum secreted molecules. Int. J. Food Microbiol. 2012, 156, 255-263. [CrossRef] [PubMed]

8. Kim, N.; Kunisawa, J.; Kweon, M.N.; Eog Ji, G.; Kiyono, H. Oral feeding of Bifidobacterium bifidum (BGN4) prevents CD4(+) CD45RB(high) T cell-mediated inflammatory bowel disease by inhibition of disordered $\mathrm{T}$ cell activation. Clin. Immunol. 2007, 123, 30-39. [CrossRef]

9. Philippe, D.; Heupel, E.; Blum-Sperisen, S.; Riedel, C.U. Treatment with Bifidobacterium bifidum 17 partially protects mice from Th1-driven inflammation in a chemically induced model of colitis. Int. J. Food Microbiol. 2011, 149, 45-49. [CrossRef]

10. Alard, J.; Peucelle, V.; Boutillier, D.; Breton, J.; Kuylle, S.; Pot, B.; Holowacz, S.; Grangette, C. New probiotic strains for inflammatory bowel disease management identified by combining in vitro and in vivo approaches. Benef. Microbes 2018, 9, 317-331. [CrossRef]

11. Guglielmetti, S.; Tamagnini, I.; Mora, D.; Minuzzo, M.; Scarafoni, A.; Arioli, S.; Hellman, J.; Karp, M.; Parini, C. Implication of an outer surface lipoprotein in adhesion of Bifidobacterium bifidum to Caco-2 cells. Appl. Environ. Microbiol. 2008, 74, 4695-4702. [CrossRef] [PubMed]

12. Guglielmetti, S.; Tamagnini, I.; Minuzzo, M.; Arioli, S.; Parini, C.; Comelli, E.; Mora, D. Study of the Adhesion of Bifidobacterium bifidum MIMBb75 to Human Intestinal Cell Lines. Curr. Microbiol. 2009, 59, 167-172. [CrossRef] [PubMed] 
13. Guglielmetti, S.; Mora, D.; Gschwender, M.; Popp, K. Randomised clinical trial: Bifidobacterium bifidum MIMBb75 significantly alleviates irritable bowel syndrome and improves quality of life — a double-blind, placebo-controlled study. Aliment. Pharm. Ther. 2011, 33, 1123-1132. [CrossRef] [PubMed]

14. Singh, N.; Arioli, S.; Wang, A.; Villa, C.R.; Jahani, R.; Song, Y.S.; Mora, D.; Guglielmetti, S.; Comelli, E.M. Impact of Bifidobacterium bifidum MIMBb75 on mouse intestinal microorganisms. FEMS Microbiol. Ecol. 2013, 85, 369-375. [CrossRef] [PubMed]

15. Taibi, A.; Singh, N.; Chen, J.; Arioli, S.; Guglielmetti, S.; Comelli, E.M. Time- and strain-specific downregulation of intestinal EPAS1 via miR-148a by Bifidobacterium bifidum. Mol. Nutr. Food Res. $2017,61$. [CrossRef] [PubMed]

16. Guglielmetti, S.; Zanoni, I.; Balzaretti, S.; Miriani, M.; Taverniti, V.; De Noni, I.; Presti, I.; Stuknyte, M.; Scarafoni, A.; Arioli, S.; et al. Murein Lytic Enzyme TgaA of Bifidobacterium bifidum MIMBb75 Modulates Dendritic Cell Maturation through Its Cysteine- and Histidine-Dependent Amidohydrolase/Peptidase (CHAP) Amidase Domain. Appl. Environ. Microbiol. 2014, 80, 5170-5177. [CrossRef] [PubMed]

17. Xue, X.; Ramakrishnan, S.; Anderson, E.; Taylor, M.; Zimmermann, E.M.; Spence, J.R.; Huang, S.; Greenson, J.K.; Shah, Y.M. Endothelial PAS domain protein 1 activates the inflammatory response in the intestinal epithelium to promote colitis in mice. Gastroenterology 2013, 145, 831-841. [CrossRef]

18. Collins, J.W.; Keeney, K.M.; Crepin, V.F.; Rathinam, V.A.; Fitzgerald, K.A.; Finlay, B.B.; Frankel, G. Citrobacter rodentium: Infection, inflammation and the microbiota. Nat. Rev. Microbiol. 2014, 12, 612-623. [CrossRef]

19. Johnson-Henry, K.C.; Nadjafi, M.; Avitzur, Y.; Mitchell, D.J.; Ngan, B.Y.; Galindo-Mata, E.; Jones, N.L.; Sherman, P.M. Amelioration of the effects of Citrobacter rodentium infection in mice by pretreatment with probiotics. J. Infect. Dis. 2005, 191, 2106-2117. [CrossRef]

20. Gareau, M.G.; Wine, E.; Reardon, C.; Sherman, P.M. Probiotics prevent death caused by Citrobacter rodentium infection in neonatal mice. J. Infect. Dis. 2010, 201, 81-91. [CrossRef]

21. Rodrigues, D.M.; Sousa, A.J.; Johnson-Henry, K.C.; Sherman, P.M.; Gareau, M.G. Probiotics Are Effective for the Prevention and Treatment of Citrobacter rodentium-Induced Colitis in Mice. J. Infect. Dis. 2012, 206, 99-109. [CrossRef] [PubMed]

22. Collins, J.W.; Chervaux, C.; Raymond, B.; Derrien, M.; Brazeilles, R.; Kosta, A.; Chambaud, I.; Crepin, V.F.; Frankel, G. Fermented dairy products modulate Citrobacter rodentium-induced colonic hyperplasia. J. Infect. Dis. 2014, 210, 1029-1041. [CrossRef] [PubMed]

23. Chen, C.C.; Chiu, C.H.; Lin, T.Y.; Shi, H.N.; Walker, W.A. Effect of probiotics Lactobacillus acidophilus on Citrobacter rodentium colitis: The role of dendritic cells. Pediatr. Res. 2009, 65, 169-175. [CrossRef] [PubMed]

24. Fanning, S.; Hall, L.J.; Cronin, M.; Zomer, A.; MacSharry, J.; Goulding, D.; Motherway, M.O.; Shanahan, F.; Nally, K.; Dougan, G.; et al. Bifidobacterial surface-exopolysaccharide facilitates commensal-host interaction through immune modulation and pathogen protection. Proc. Natl. Acad. Sci. USA 2012, 109, 2108-2113. [CrossRef] [PubMed]

25. Collins, J.W.; Akin, A.R.; Kosta, A.; Zhang, N.; Tangney, M.; Francis, K.P.; Frankel, G. Pre-treatment with Bifidobacterium breve UCC2003 modulates Citrobacter rodentium-induced colonic inflammation and organ specificity. Microbiology 2012, 158, 2826-2834. [CrossRef]

26. Bhinder, G.; Sham, H.P.; Chan, J.M.; Morampudi, V.; Jacobson, K.; Vallance, B.A. The Citrobacter rodentium mouse model: Studying pathogen and host contributions to infectious colitis. J. Vis. Exp. 2013, e50222. [CrossRef]

27. Wiles, S.; Clare, S.; Harker, J.; Huett, A.; Young, D.; Dougan, G.; Frankel, G. Organ specificity, colonization and clearance dynamics in vivo following oral challenges with the murine pathogen Citrobacter rodentium. Cell. Microbiol. 2004, 6, 963-972. [CrossRef]

28. Wiles, S.; Pickard, K.M.; Peng, K.; MacDonald, T.T.; Frankel, G. In vivo bioluminescence imaging of the murine pathogen Citrobacter rodentium. Infect. Immun. 2006, 74, 5391-5396. [CrossRef]

29. Wen, B.; Tokar, T.; Taibi, A.; Chen, J.; Jurisica, I.; Comelli, E.M. Citrobacter rodentium alters the mouse colonic miRNome. Genes immune. 2018. [CrossRef]

30. Glenn, A.J.; Fielding, K.A.; Chen, J.; Comelli, E.M.; Ward, W.E. Long-term vitamin D3 supplementation does not prevent colonic inflammation or modulate bone health in IL-10 knockout mice at young adulthood. Nutrients 2014, 6, 3847-3862. [CrossRef] 
31. Lupp, C.; Robertson, M.L.; Wickham, M.E.; Sekirov, I.; Champion, O.L.; Gaynor, E.C.; Finlay, B.B. Host-mediated inflammation disrupts the intestinal microbiota and promotes the overgrowth of Enterobacteriaceae. Cell Host Microbe 2007, 2, 119-129. [CrossRef] [PubMed]

32. Kainulainen, V.; Reunanen, J.; Hiippala, K.; Guglielmetti, S.; Vesterlund, S.; Palva, A.; Satokari, R. BopA Does Not Have a Major Role in the Adhesion of Bifidobacterium bifidum to Intestinal Epithelial Cells, Extracellular Matrix Proteins, and Mucus. Appl. Environ. Microbiol. 2013, 79, 6989-6997. [CrossRef] [PubMed]

33. Foroni, E.; Serafini, F.; Amidani, D.; Turroni, F.; He, F.; Bottacini, F.; Motherway, M.O.; Viappiani, A.; Zhang, Z.D.; Rivetti, C.; et al. Genetic analysis and morphological identification of pilus-like structures in members of the genus Bifidobacterium. Microb. Cell Fact. 2011, 10. [CrossRef] [PubMed]

34. Hill, C.; Guarner, F.; Reid, G.; Gibson, G.R.; Merenstein, D.J.; Pot, B.; Morelli, L.; Canani, R.B.; Flint, H.J.; Salminen, S.; et al. The International Scientific Association for Probiotics and Prebiotics consensus statement on the scope and appropriate use of the term probiotic. Nat. Rev. Gastroenterol. Hepatol. 2014, 11, 506-514. [CrossRef] [PubMed]

35. Teitelbaum, A.A.; Gareau, M.G.; Jury, J.; Yang, P.C.; Perdue, M.H. Chronic peripheral administration of corticotropin-releasing factor causes colonic barrier dysfunction similar to psychological stress. Am. J. Physiol. Gastrointest. Liver Physiol. 2008, 295, G452-G459. [CrossRef] [PubMed]

36. FAO/WHO. Joint FAO/WHO Working Group Report on Drafting Guidelines for the Evaluation of Probiotics in Food. London, Ontario, Canada, 30 April-1 May 2002. Available online: http:/ / www.fao.org/3/a-a0512e. pdf (accessed on 11 February 2019).

37. Abraham, B.P.; Quigley, E.M.M. A Probiotic for Ulcerative Colitis: The Culture Wars Continue. Dig. Dis. Sci. 2018, 63, 1678-1680. [CrossRef] [PubMed]

(C) 2019 by the authors. Licensee MDPI, Basel, Switzerland. This article is an open access article distributed under the terms and conditions of the Creative Commons Attribution (CC BY) license (http:/ / creativecommons.org/licenses/by/4.0/). 